\title{
Molecular hydrogen inhalation prevents L-DOPA-induced dyskinesia in a Parkinson's disease rat model
}

\author{
Glauce Nascimento ${ }^{1}$ and Elaine Del Bel ${ }^{1}$
}

${ }^{1}$ Affiliation not available

February 15, 2022 

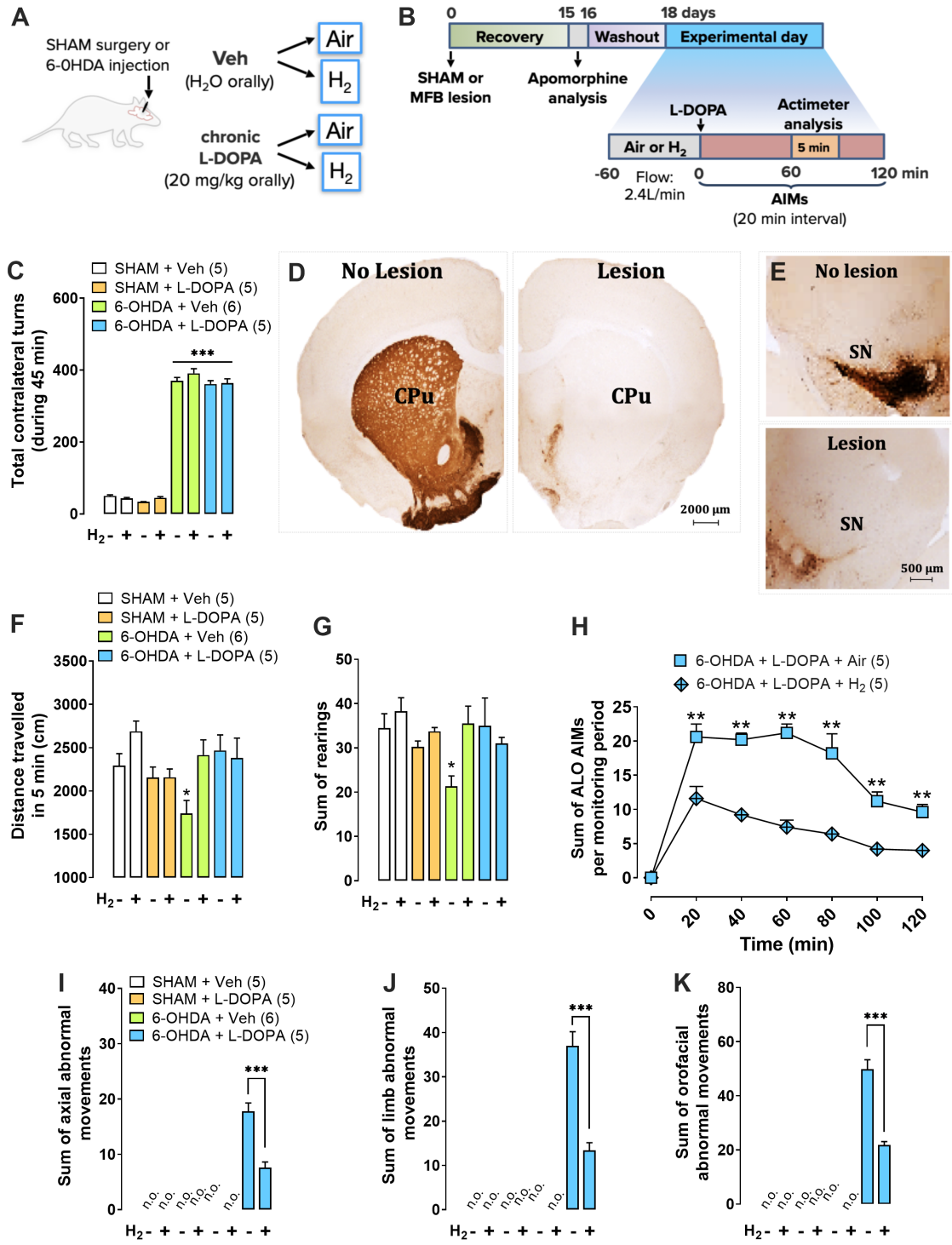

Figure 1: This is a Figure 1. Molecular hydrogen $\left(\mathbf{H}_{\mathbf{2}}\right)$ inhalation reduces AIMS without interfering with the anti-parkinsonian effect of L-DOPA. (A, B) Schematic protocol. After Sham surgery or 6-OHDA injection, the rats were analysed in the apomorphine-induced rotational test for lesion confirmation. Then, Sham or 6-OHDA-lesioned rats were treated chronically (15 days) with L-DOPA or its vehicle. Subsequently before the last L-DOPA injection, Air mixture or $\mathrm{H}_{2}$ inhalation was performed for a 1-h period, followed by the L-DOPA injection and abnormal involuntary movements on axial, limb and orofacial parameters (ALO AIMs) and locomotor activity were measured. (C) 6-OHDA lesion significantly increased total contralateral turns induced by apomorphine. (D) Photomicrographs of coronal brain sections illustrating the loss of $\mathrm{TH}$ positive immunolabeling in the striatum fibers (D) and substantia nigra compacta neurons (E), scale bars $=1200 \mu \mathrm{m}$. (F, G) $\mathrm{H}_{2}$ treatment does not alter the L-DOPA effect on the distance traveled $(\mathrm{F})$ and rearing $(\mathrm{G})$ in the actimeter. $(\mathrm{H})$ Time course of the appearance of dyskinetic manifestation with 6-OHDA+L-DOPA+Air mixture compared to 6-OHDA+L-DOPA $+\mathrm{H}_{2}$ inhalation $(\mathrm{p}<0.001)$ across time (for each 20-min period). (I, J, K) show the co-administration of L-DOPA and $\mathrm{H}_{2}$ inhalation effects on the axial (I), limb (J) and orofacial (K) AIMs over 120 mins. Sham rats treated with vehicle of L-DOPA (SHAM+Veh, white); Sham rats treated chronically with L-DOPA (SHAM+L-DOPA, orange); 6-OHDA lesioned rats treated with vehicle of L-DOPA (6-OHDA+Veh, green); 6-OHDA lesioned rats treated chronically with L-DOPA (6-OHDA+L-DOPA, blue). AIMs were analyzed using 2-Way RM ANOVA analysis with Tukey's multiple comparisons post-hoc test. Distance travelled was analyzed by ordinary One-Way ANOVA with Fisher post-hoc test. Data are reported as mean \pm SEM. ${ }^{* * *} \mathrm{p}<0.001,{ }^{* *} \mathrm{p}<0.01,{ }^{*} \mathrm{p}<0.05$. caption 

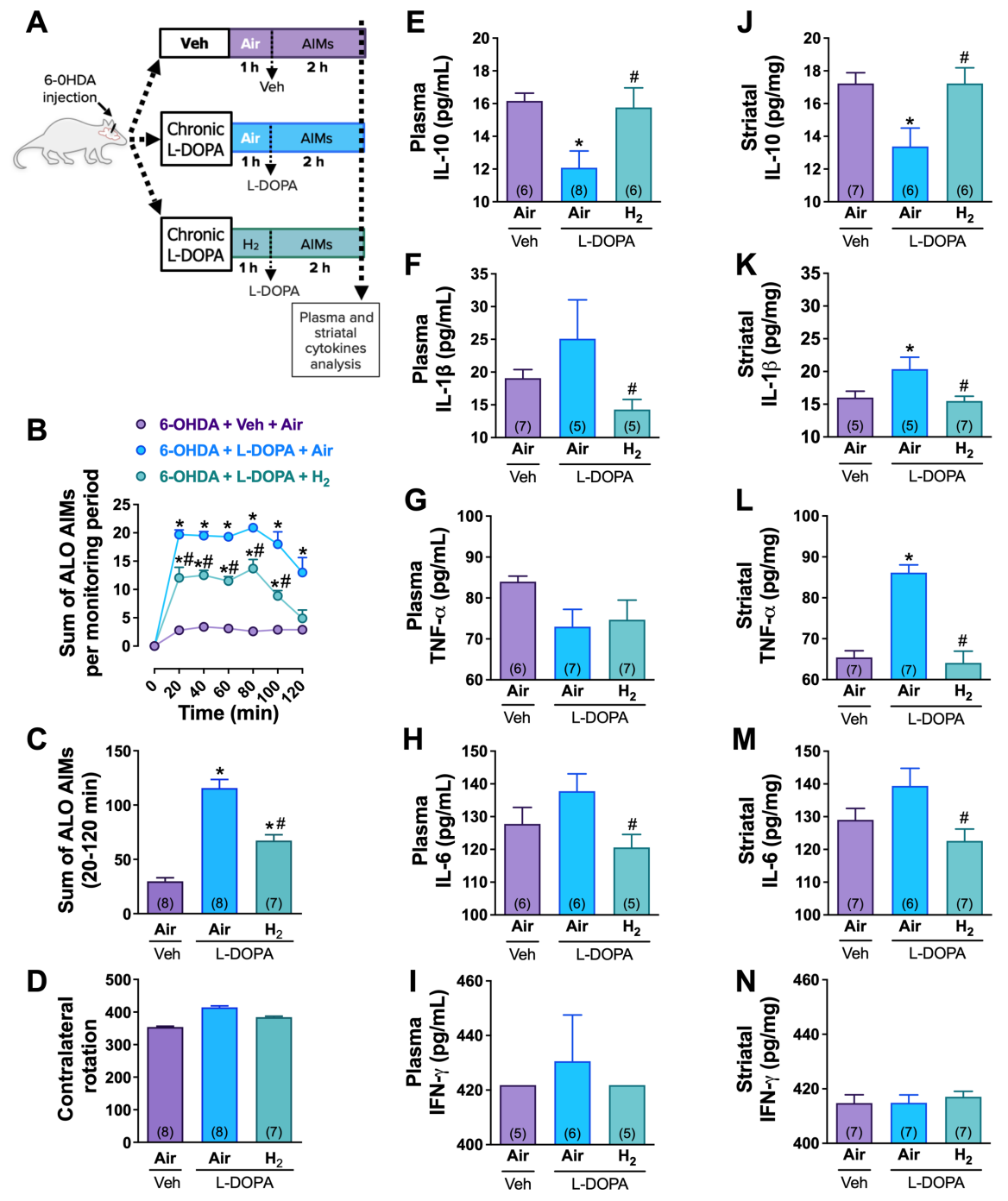

Figure 2: This is Figure 2. Molecular hydrogen $\left(\mathbf{H}_{2}\right)$ inhalation improves peripheral and striatal inflammation in L-DOPA-treated 6-OHDA-lesioned rats. (A) Schematic timeline for cytokine measurements. (B) Time course of the appearance of dyskinetic manifestation of the animals used for cytokines measurement (6-OHDA+Veh+Air mixture; 6-OHDA+L-DOPA+Air mixture; 6-OHDA+L-DOPA+ $\mathrm{H}_{2}$ inhalation; $\mathrm{p}<0.001$ ) across time (for each 20-min period). (C) show the co-administration of L-DOPA and $\mathrm{H}_{2}$ inhalation effects on the sum of axial, limb and orofacial AIMs over 120 mins of these animals. (D) These animals were tested also in the apomorphine test, 6-OHDA lesion significantly increased total contralateral turns induced by apomorphine. Plasma and striatal levels of IL-10 (E and J), IL-1 $\beta$ (F and K), TNF- $\alpha$ $(\mathrm{G}$ and $\mathrm{L})$, IL-6 ( $\mathrm{H}$ and $\mathrm{M})$ and IFN- $\gamma$ (I and N) 2 hours after L-DOPA administration in rats inhaling $\mathrm{H}_{2}$ (L-DOPA $+\mathrm{H}_{2}$ ) or not (L-DOPA + Air). 6-OHDA-lesioned rats were represented by the purple bars; 6-OHDA-lesioned rats treated chronically with L-DOPA inhaling Air were represented by the blue bars, and 6-OHDA-lesioned rats treated chronically with L-DOPA and inhaling $\mathrm{H}_{2}$ were represented by the green bars. $\mathrm{H}_{2}$ inhalation caused an increase in plasma and striatal levels of IL-10 $(\mathrm{p}<0.05)$ and a decrease in plasma and striatal levels of IL- $1 \beta(\mathrm{p}<0.05)$, striatal levels of TNF- $\alpha(\mathrm{p}<0.05)$ and plasma and striatal levels of IL-6 $(\mathrm{p}<0.05)$ in L-DOPA treated rats. All cytokine measurements were statistically analyzed by ordinary One-Way ANOVA with Fisher's post-hoc test. Bars are represented as mean \pm SEM. \# p $<0.05$ vs. L-DOPA + Air (blue bars), ${ }^{*}$ p $<0.05$ vs. Veh + Air (purple bars) groups. a caption 

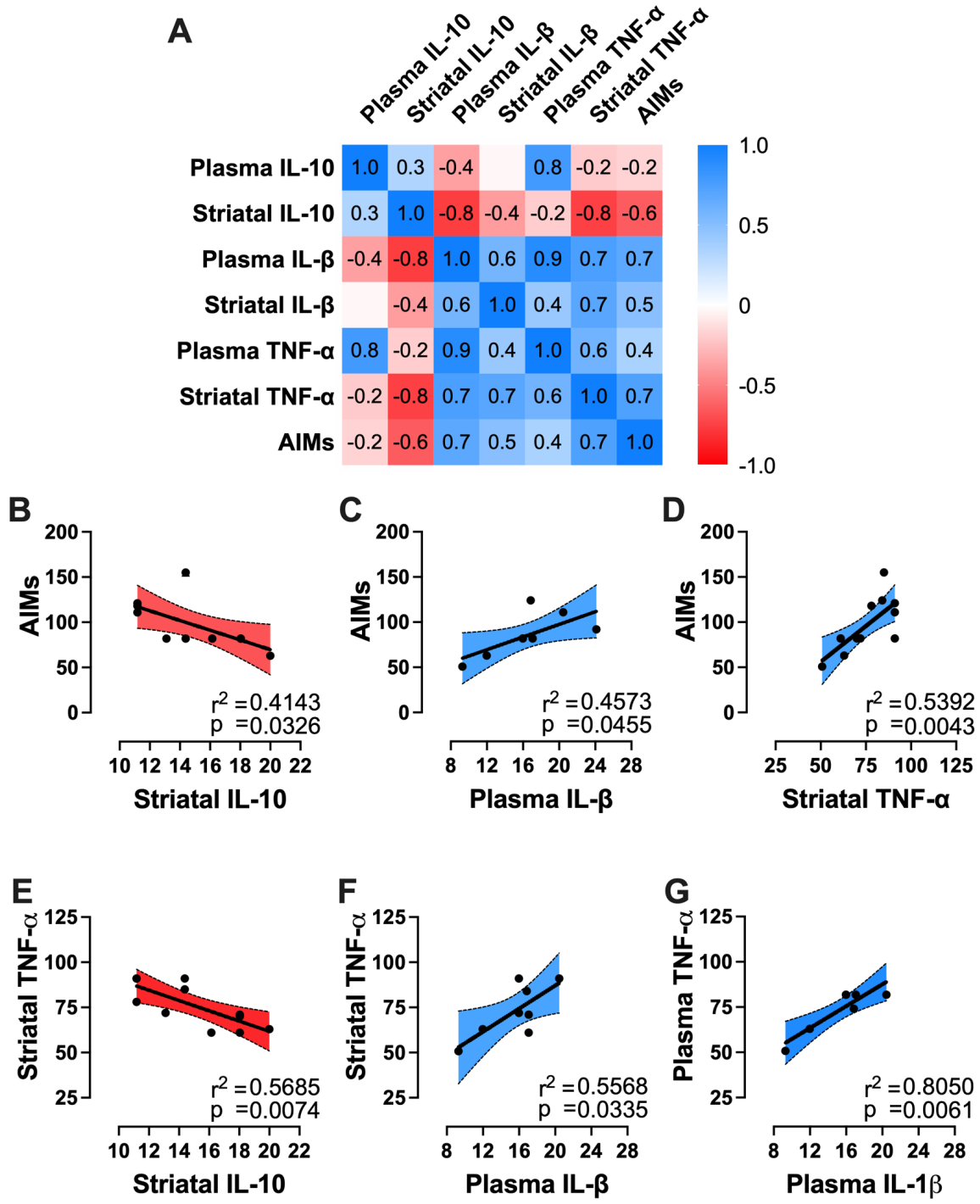

Figure 3: This is Figure 3. AIMS were negatively correlated with striatal IL-10 and positively correlated with plasma IL-1 $\beta$ and striatal TNF- $\alpha$ levels.(A) Correlation matrix of plasma and striatal IL-10, IL-1 $\beta$, TNF- $\alpha$ levels, and AIMs. The correlation coefficient ( $r$ ) of each correlation is inside its correspondent box ranging from -1 (darkest red gradient) to +1 (darkest blue gradient). A two-tailed p-value was chosen to evaluate the statistical significance of each correlation. The coefficient of determination $\left(\mathrm{r}^{2}\right)$ between a statistically correlated pair of variables was calculated and a best-fit linear regression line (dark full line in the plot) was built for each two variables in one panel (panels B-G). Correlation plot was set at 95\% confidence interval (shadowed area with the exactly gradient of the correspondent correlation coefficient - r) surrounded by dark dashed lines. There was a statistical correlation between: AIMS and striatal IL-10 levels (B); AIMS with plasma IL-1 $\beta$ (C) and AIMs with striatal TNF- $\alpha$ (D) levels; striatal TNF- $\alpha$ levels with striatal IL-10 (E), plasma IL-1 $\beta$ with striatal TNF- $\alpha(\mathrm{F})$, and plasma TNF- $\alpha(\mathrm{G})$ levels. a caption 

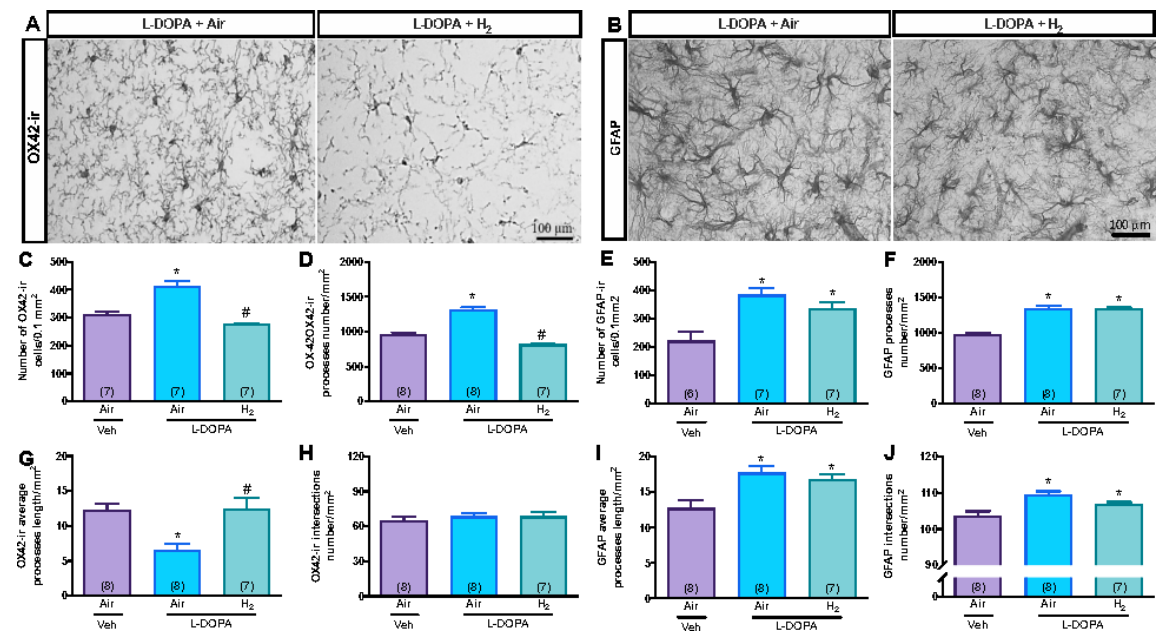

Figure 4: This is a cap Figure 4. Molecular hydrogen $\left(\mathbf{H}_{\mathbf{2}}\right)$ inhalation prevented the reactivity of striatal microglia with no impact in striatal astrocytes morphology. Microglia analysis by OX42-ir staining. (A) representative photomicrographs of striatum slices of lesioned rats treated chronically with L-DOPA inhaling (L-DOPA $+\mathrm{H}_{2}$ ) or not (L-DOPA) $\mathrm{H}_{2}$. Quantification of OX-42-ir images indicates the number of OX-42-ir cells (B), total number of processes cells (C), average processes length (D), and intersections number between the processes $(\mathrm{G})$. $\mathrm{H}_{2}$ treatment induced a decrease in the number of OX42-ir positive cells, in the average of processes number and length, a without affecting the number of intersections in L-DOPA-treated rats (Fig. H; p> 0.05). Astrocyte analysis by GFAP staining. (B) representative photomicrographs of striatum slices of 6-OHDA-lesioned rats treated chronically with L-DOPA inhaling (L-DOPA + $\mathrm{H}_{2}$ ) or not (L-DOPA) $\mathrm{H}_{2}$. Quantification of GFAP images indicates the number of GFAP cells (E), the total number of processes cells (F), average processes length (I), and intersections number between the processes $(\mathrm{J}) . \mathrm{H}_{2}$ treatment caused no alterations in the number of GFP positive cells, processes number, length, and intersections $(\mathrm{p}>0.05)$. 6-OHDA-lesioned rats were represented by the purple bars; 6-OHDA-lesioned rats treated chronically with L-DOPA inhaling Air was represented by the blue bars, and 6-OHDA-lesioned rats treated chronically with L-DOPA and inhaling $\mathrm{H}_{2}$ were represented by the green bars. Data were statistically analyzed by ordinary One-Way ANOVA with Fisher post-hoc test. Bars are represented as mean \pm SEM. $\#$ p $<0.05$ vs. L-DOPA + Air, ${ }^{*}$ p $<0.05$ vs. Veh + Air group tion

Bruna M. Santos $\mathrm{PhD}^{1,2}$; Glauce C. Nascimento $\mathrm{PhD}^{1,3}$; João F. Pedrazzi $\mathrm{PhD}^{4}$; Danyelle Silva-Amaral $\mathrm{MS}^{1}$; Mariza Bortolanza $\mathrm{PhD}^{3}$; Grant T. Harris $\mathrm{BS}^{2}$; Luiz G. S. Branco PhD ${ }^{1,3 *}$, Elaine Del Bel PhD ${ }^{1,3,4 *}$

${ }^{1}$ Department of Physiology, Medical School of Ribeirão Preto, University of São Paulo, Ribeirão Preto, SP, Brazil.

${ }^{2}$ Thermoregulation and Systemic Inflammation Laboratory (FeverLab), St. Josephs' Hospital and Medical Center, Phoenix, AZ, USA.

${ }^{3}$ Department of Basic and Oral Biology, Ribeirão Preto Dentistry Faculty, University of São Paulo, Ribeirão Preto, SP, Brazil.

${ }^{4}$ Neuroscience Graduate Program, Medical School of Ribeirão Preto, University of São Paulo, Ribeirão Preto, SP, Brazil.

*Corresponding Authors :

Elaine Del Bel ; email:eadelbel@usp.br; Department of Basic and Oral Biology, Dental School, University of São Paulo (USP), Ribeirão Preto, SP, 14040-904, Brazil. 
Luiz GS Branco ; email: branco@forp.usp.br ; Department of Basic and Oral Biology, Dental School, University of São Paulo (USP), Ribeirão Preto, SP, 14040-904, Brazil.

Text (3692 words)- (Up to 3700 words excluding of abstract, legends, and references)

Running title: The anti-inflammatory effect of $\mathrm{H}_{2}$ in LID

Key words: 6-Hydroxydopamine, Parkinson's disease, neuroinflammation; striatum; systemic inflammation

The authors declare that the research was conducted in the absence of any commercial or financial relationships that could be construed as a potential conflict of interest.

Funding sources: Grant GCN [\#88882.317597/2019-014, National Council for Scientific and Technological Development (PNPD-CNPq), Brazil]. Grant JFP [\#88887.341956/2019-00, Programa Nacional de Pósdoutorado, Coordenação de Aperfeiçoamento de Pessoal de Nível Superior (PNPD/CAPES)]. Grant MB [\#88887.334730/2019-00, Coordenação de Aperfeiçoamento de Pessoal de Nível Superior (CAPES)]. Grant LGSB [\#2016/17681-9, São Paulo Research Foundation (FAPESP), Brazil. Grant EDB [\#2017/2430440, São Paulo Research Foundation (FAPESP), Brazil]; [\# 88881.198836/2018/01, Coordenação de Aperfeiçoamento de Pessoal de Nível Superior and Deutscher Akademischer Austauschdienst (CAPES/DAAD - 2019-2024), Brazil and Germain], [\#88887.192409/2018-01, Coordenação de Aperfeiçoamento de Pessoal de Nível Superior and Comitê Francês de Avaliação da Cooperação Universitária com o Brasil (CAPES/COFECUB- 2019-2024), Brazil and France.], [\#302434/2019-04, National Council for Scientific and Technological Development (CNPq), Brazil].

Abstract (226 words)

Background: L-3,4-dihydroxyphenylalanine (L-DOPA)-induced dyskinesia is a side effect of Parkinson's disease treatment and it is characterized by atypical involuntary movements. A link between neuroinflammation and L-DOPA-induced dyskinesia has been documented. Molecular hydrogen has neuroprotective effects in Parkinson's disease models and has a major anti-inflammatory effect.

Objective: To test the hypothesis that molecular hydrogen inhalation reduces L-DOPA-induced dyskinesia.

Methods: 15 days after 6-hydroxydopamine lesions of dopaminergic neurons were made (microinjection into the medial forebrain bundle), chronic L-DOPA treatment (15 days) was performed. Rats were exposed to molecular hydrogen ( $2 \%$ gas mixture, $1 \mathrm{~h}$ ) or air (controls) before L-DOPA injection. Abnormal involuntary movements and locomotor activity were conducted. Striatal and plasma samples for molecular and morphological evaluations were collected after the abnormal involuntary movements analysis.

Results: Molecular hydrogen inhalation attenuated L-DOPA-induced dyskinesia. The gas therapy did not impair the improvement of locomotor activity achieved by L-DOPA treatment. Display of abnormal involuntary movements was positively correlated with plasma IL- $1 \beta$ and striatal TNF- $\alpha$ levels and negatively correlated with striatal IL-10 levels. $\mathrm{H}_{2}$ inhalation reduced activated microglia in the lesioned striatum, which is consistent with the observed reduced pro-inflammatory cytokines levels.

Conclusions and implications: Prophylactic molecular hydrogen inhalation decreases abnormal involuntary movements in a preclinical L-DOPA-induced dyskinesia model. The molecular hydrogen antidyskinetic effect was associated with decreased striatal and peripheral inflammation. This finding has a translational importance to L-DOPA-treated parkinsonian patients' well-being.

\section{Introduction}

Several motor and non-motor debilitating symptoms of Parkinson's disease (PD) are generated by dopamine (DA) depletion. Levodopa (L-DOPA) is recognized as the most effective and well tolerated drug for PD treatment. However, long-term L-DOPA therapy has been associated with the development of abnormal involuntary movements referred to as L-DOPA-induced dyskinesia (LID) ${ }^{1}$. The development of dyskinesia represents a serious side effect that negatively affects quality of life of people with $\mathrm{PD}$, and is also associated with both motor, including an increased risk of postural imbalance and trauma from falls, and non-motor 
operations, particularly, fluctuations in mood and cognitive performance ${ }^{2-4}$. Although various compounds have been used for treating LID, their success has been limited ${ }^{3}$.

The pathogenesis of LID is associated with non-physiological stimulation of DA receptors using L-DOPA, which combined with the reduction of gamma-aminobutyric acid transmission pale-thalamic activity, generates neuronal hyperactivity in the striatum ${ }^{5-9}$. Moreover, recent studies suggest that astrocyte and microglia activation increase pro-inflammatory cytokines production, contributing to the appearance of LID ${ }^{10,11}$. In addition, excessive levels of glutamate and dopamine in the striatum feed this inflammatory environment, favoring LID ${ }^{12,13}$. A key unmet medical need for the PD community is a strategy for ameliorating $\operatorname{LID}^{10,14,15}$.

Despite the fact that neurodegenerative disorders and associated consequences have been consistently related to central nervous system (CNS) derangement, they also have a strong associative component with environmental factors ${ }^{16}$. Given the evidence of the profound influence of the periphery producing environmental signals to the CNS, the periphery-brain axis has been increasingly explored within the neurodegenerative disorders such as Alzheimer's and $\mathrm{PD}^{17,18}$. One of the peripheral influences that has been implicated in the neurodegenerative disorders outbreak and progression is the peripheral immune system ${ }^{19}$. Intestinal inflammation, for example, is remarkably observed in patients with $\mathrm{PD}^{20,21}$. Gut microbiota related to $\mathrm{PD}$ clinical phenotype produces an improper ratio of sub-products with anti-inflammatory agents such as short-chain fatty acids and molecular hydrogen $\left(\mathrm{H}_{2}\right)^{22,23}$.

Studies have shown that $\mathrm{H}_{2}$ may protect the brain from various neuronal conditions, including neurological emergencies and neurodegenerative diseases ${ }^{24-26} \cdot \mathrm{H}_{2}$ in rodents protects mesencephalic dopaminergic neurons from 1-methyl-4-phenyl-1,2,3,6-tetrahydropyridine (MTPT) ${ }^{24}$ and 6-hydroxydopamine (6-OHDA ${ }^{25}$ induced degeneration. Several lines of evidence indicate that neuro-immune function and behavior are influenced by $\mathrm{H}_{2}{ }^{26-28}$. Accumulating studies indicate that $\mathrm{H}_{2}$ not only has antioxidant ${ }^{29,30}$ but also anti-inflammatory properties $^{31-33}$. However, there are no previous studies addressing the putative $\mathrm{H}_{2}$ benefits on LID.

Hence, we tested the hypothesis that $\mathrm{H}_{2}$ inhalation reduces L-DOPA-induced dyskinesia in an animal PD model. We evaluated the effect of $\mathrm{H}_{2}$ inhalation on abnormal involuntary movements (AIMs) in PD rats treated with L-DOPA. Moreover, we evaluated the role of $\mathrm{H}_{2}$ in pro-inflammatory [tumor necrosis factor (TNF)- $\alpha$, interleukin (IL)-1 $\beta$, IL-6, interferon (IFN)-, and anti-inflammatory (IL-10) cytokine levels in the plasma and striatum of PD-lesioned rats treated with L-DOPA. Finally, we examined whether $\mathrm{H}_{2}$ inhalation influenced the morphology of striatal astrocytes and microglia in PD-lesioned rats chronically treated with L-DOPA.

2.0. Materials and Methods More detailed description of our experimental procedures can be found in the Supplementary Methods.

2.1. Animals Male Wistar rats (200-250 g, $\mathrm{n}=65$; USP-RP, SP, Brazil) were used. The experimental protocol followed the Ethical Principles in Animal Research delineated by the guidelines for the care and use of mammals in Neuroscience and Behavioral Research and was approved by the Ethics Committee on the Use of Experimental Animals of the University of São Paulo (\#2016.1.667.58.4).

2.2. Dopaminergic lesion with neurotoxin 6-hydroxydopamine Rats were anesthetized with 2,2,2tribromoethanol $\left(250 \mathrm{mg} \mathrm{kg}^{-1} \mathrm{ip}\right)$ and fixed into the stereotaxic apparatus. The incisor bar was set at 3.3 $\mathrm{mm}$ below the interaural line. Rats received one injection of $2.0 \mu \mathrm{l}$ 6-OHDA into the left medial forebrain bundle as described by Gomes et al., $2008^{5}$ (6-OHDA - $2.5 \mu \mathrm{g} \mathrm{ul}^{-1}$ in $0.9 \% \mathrm{NaCl}$ supplemented with $0.02 \%$ ascorbic acid, $\left.1 \mu \mathrm{L} \mathrm{min}^{-1}\right)$.

Motor asymmetry was assessed 15 days after the lesion of the nigrostriatal pathway by apomorphine-induced rotational behavior analysis $(0.5 \mathrm{mg} \mathrm{kg} \text { in } 0.9 \% \mathrm{NaCl} \text {, subcutaneous, Sigma) (Figs. 1C and 2D) })^{34}$. The lesion was confirmed histologically at the end of the behavioral tests (described below) by tyrosine hydroxylase (TH) immunohistochemistry in the striatum and substantia nigra (Figs.1D and E). L-DOPA treatment started two days after the apomorphine-induced rotational test.

2.3. $\mathbf{H}_{2}$ administration All animals were adapted to the chamber with ambient air at a flow rate of 2.4 
$\mathrm{L} / \mathrm{min}$, one day before the experiments. On the experimental day, animals were exposed to $2 \% \mathrm{H}_{2}$-mixture ( $21 \%$ of $\mathrm{O}_{2}$ balanced with $\mathrm{N}_{2}$ ) or Air-mixture $\left(0 \% \mathrm{H}_{2} ; 21 \% \mathrm{O}_{2} \mathrm{~N}_{2}\right.$-balanced) for 1 hour at the same flow rate as the training day. Continuous monitoring of $\mathrm{H}_{2}$ concentration in the chamber was made possible by using a gas analyzer to verify the $\mathrm{H}_{2}$ outflow from the chamber. The tested concentration of $\mathrm{H}_{2}$ in the mixture $(2 \%)$ was chosen due to the consistent and repeatable anti-inflammatory effect observed in previous studies $^{29,32,33}$.

2.4. L-DOPA-induced abnormal involuntary movements analysisRats were subjected to chronic L-DOPA (20 mg kg plus benserazide $5 \mathrm{mg} \mathrm{kg}$, orally by gavage) administration for 15 days (once a day) to induce a stable expression of dyskinesia ${ }^{14}$. Animals showing AIMs scores greater than 10 and severity grading greater than 2 on at least one AIMs subtype during the effect of L-DOPA were selected for the behavioral study $^{30-32}$. Rats were monitored individually for orofacial and limb dyskinesia, and axial dystonia or AIMs using a rat dyskinesia scale ${ }^{34-37}$. This scale considers the severity and amplitude of the axial, limb and orolingual AIMs (the scores ranged from 0 to 4) (for review see Cenci and Lundblad $2007^{38}$ ). A dyskinesia time curve was generated by plotting global AIMs scores from each monitoring time individually for an entire testing session (180 min).

2.5. Actimeter test To evaluate possible motor effects of the treatments, the spontaneous locomotor activity of each animal was individually assessed in a photoelectric actimeter (Actitrack, Panlab, Barcelona, Spain). The apparatus consists of a square arena $(45 \times 45 \times 20 \mathrm{~cm})$ equipped with 16 photocells that detect horizontal movement and 16 photocells that detect vertical movement. Thus, the locomotor activity was obtained from recording the number of interruptions of the photoelectric cells during 5 minutes. The amount of rearings was counted using total vertical activity movements.

2.6. Plasma and striatum sampling The animals were euthanized by decapitation 1 hour after LDOPA or its vehicle administration. The trunk blood was rapidly collected in heparin-coated tubes and subsequently centrifuged at $3,500 \mathrm{rpm}$ for $20 \mathrm{~min}$ at $4^{\circ} \mathrm{C}$. The brain was removed, and the rostral and medial portion of the striatum (rostral: $1.7 \mathrm{~mm}$ and medial: $0.7 \mathrm{~mm}$ from bregma) was carefully excised, frozen by submersion in dry ice chilled isopentane and The medial-caudal (medial: $0.7 \mathrm{~mm}$ and caudal: $-0.8 \mathrm{~mm}$ and from bregma) portion of the striatum was collected and post-fixed in tamponade paraformaldehyde (4\%) for immunohistochemistry preparation.

2.7. Measurement of plasma and striatal cytokines levels The inflammatory profile of the target samples was evaluated using a high sensitivity enzyme-linked immunosorbent assay (Luminex). Luminex assay was conducted using Luminex Magpix technology (Austin, TX, USA) according to manufacturer guidelines (LXSARM - 05, R\&D, Minnesota, MN, USA) ${ }^{39}$.

2.8. Immunohistochemistry Serial coronal sections throughout the mediocaudal extent of the striatum (Bregma $+2.76 \mathrm{~mm}$, Interaural $11.76 \mathrm{~mm}-$ Bregma $-2.28 \mathrm{~mm}$, Interaural $6.72 \mathrm{~mm}$ ) were cut $(25 \mu \mathrm{m})$ using a freezing microtome (Leica, model CM1850). Immunohistochemistry was performed in these sections using a standard peroxidase-based method ${ }^{5}$ to quantify TH, glial fibrillary acidic protein (GFAP) and OX-42 (CD11b/c equivalent protein of microglia). The sections were incubated overnight at room temperature with the primary antibodies (Suppl. Table 1): anti-TH; anti-GFAP or anti-OX-42, followed by $2 \mathrm{~h}$ of incubation with biotinylated secondary antibody (1:250, Vectastain). The chromogen used was diaminobenzidine (Sigma-Aldrich, St. Louis, MO, USA) and the slices were mounted on slides and cover slipped for microscopic observations. Digital images were obtained using a Leica microscope (Leica Microsystems Launches Leica FW4000 - Cambridge, UK) under 20x (for TH optical density and OX-42/GFAP number of cells quantification) or 40x (for OX-42/GFAP morphology) objectives. The quantification of the brain area was measured using the ImageJ system (ImageJ, RRID: SCR_003070,National Institutes of Health - NIH; Schneider et al., $2012^{40}$ ). Analysis of microglia (OX-42) and astrocyte (GFAP) immune labeling morphology was conducted as described by Giocanti-Auregan et al. $(2016)^{41}$ using Fiji algorithms (RRID: SCR_002285) and a generated skeleton image. The parameters analyzed were the number of cells (per $0.1 \mathrm{~mm}^{2}$ ), branches, the number of intersections or branching points, and the mean process length ${ }^{42}$. 


\subsection{Experimental design 1: effects of $\mathbf{H}_{2}$ inhalation on AIMs.}

Four experimental groups were randomly established consisting of animals that underwent SHAM surgery or medial forebrain bundle lesion with daily L-DOPA or vehicle treatment during 15 days. These groups were subdivided into animals that inhaled Air mixture or $\mathrm{H}_{2}$ gas during 1 hour on the experimental day. One day before, all the animals were adapted to the air chamber.

On the experimental day, L-DOPA or its vehicle was injected immediately after gas inhalation. AIMs analysis was conducted immediately after L-DOPA or its vehicle administration during 120 minutes. Distance travelled and amount of rearing performed by the animals were evaluated 60 minutes after L-DOPA injection for 5 minutes using the actimeter apparatus. The animals were euthanized at the end of the AIMs analysis.

2.10. Experimental design 2: effect of $\mathrm{H}_{2}$ inhalation on peripheral and striatal inflammatory profile. After a successful dopaminergic lesion and subsequent L-DOPA treatment for 15 days, the animals adapted to the air chamber one day before the experimental day.

Three experimental groups were arranged with randomized rats as follows: (i) 6-OHDA lesioned rats with L-DOPA vehicle treatment and Air mixture inhalation; (ii) 6-OHDA lesioned rats with chronic L-DOPA treatment inhaling the Air mixture; (iii) 6-OHDA lesioned rats with chronic L-DOPA treatment inhaling $\mathrm{H}_{2}$ gas.

On the experimental day, the animals were exposed to the air or $\mathrm{H}_{2}$ gas mixture for 1 hour, and L-DOPA or its vehicle was injected immediately after gas inhalation. AIMs analysis was conducted immediately after L-DOPA or its vehicle administration. The striatum and plasma were collected 2 hours after L-DOPA injection. The molecular evaluations were conducted following the protocol mentioned previously.

2.11. Statistical Analysis Parametrics statistics were performed in AIMs analysis since the basic scores of LID correspond to the extent of time with behavioral responses development ${ }^{38}$. AIMs analysis along the time were assessed by Two Way Repeated Measures Anova with Tukey's multiple comparisons post-hoc test. Ordinary One Way Anova with Fisher post-hoc test was conducted to analyze the other experiments. Statistical analysis of these experiments was performed with the significance set at $\mathrm{p}<0.05$. Correlations between cytokine levels and AIMs in L-DOPA-treated rats were analyzed by mean Pearson correlation coefficients (two-tailed).

\subsection{Results}

3.1. General effect of $\mathbf{H}_{2}$ on locomotor activity and AIMs. Only rats demonstrating more than 80 contralateral turns after the apomorphine challenge were used in this study to compound 6-OHDA groups (Fig.1C). TH-immunoreactivity was examined in coronal sections of the striatum and substantia nigra of parkinsonian animals to confirm the total lesion induced by 6-OHDA (Fig. 1D and E, respectively). Rats with the 6-OHDA lesions exhibited more than $90 \%$ reduction in TH-immunostaining in the striatum and substantia nigra compacta compared with non-lesioned animals (Fig. 1D and E). About locomotor activity, 6-OHDA lesion induced a reduction of distance traveled and rearing compared to SHAM rats and 6-OHDA lesioned rats treated with L-DOPA or $\mathrm{H}_{2}(\mathrm{p}<0.05$; Fig. $1 \mathrm{~F}$ and $\mathrm{G})$. Curiously, $\mathrm{H}_{2}$ inhalation induced an increase in locomotor activities of 6-OHDA lesioned rats treated with L-DOPA vehicle compared with those inhaling air mixture, indicating its beneficial effect per se. As previously described ${ }^{3}$, we observed an increase in distance travelled (Fig. 1F) and rearing (Fig. 1G) in L-DOPA-treated lesioned animals compared to 6-OHDA lesioned animals. The total distance travelled and rearing behavior were similar in both groups under L-DOPA administration, with Air mixture and $\mathrm{H}_{2}$ inhalations, indicating that $\mathrm{H}_{2}$ inhalation did not impair the L-DOPA benefits to restore movement.

The AIMs quantification elucidated the $\mathrm{H}_{2}$ anti-dyskinetic effect. Individual curves for each L-DOPA induced AIMs score category (limb, orofacial, and axial) were detected and it was observed an increase of AIMs in all of them ( $\mathrm{p}<0.05$, Fig. 1I-K) in 6-OHDA lesioned rats treated chronically with L-DOPA. $\mathrm{H}_{2}$ inhalation produced a reduction of $48 \%$ in LID (Figs. 1I-K). SHAM groups present no dyskinesia. The maximum total 
AIMs score reached 20-80 min after L-DOPA administration and gradually declined to the baseline level over 120 min (Figs. $1 \mathrm{H}$ and $2 \mathrm{C}$ ).

3.2. Effect of $\mathbf{H}_{2}$ in plasma and striatal inflammatory profile of LID rats The rats used for Experiment 2 were confirmed as to the intensity of the lesion and dyskinesia (Fig. 2 B-D). The apomorphineinduced rotations test confirmed the presence of lesion in all rats (Fig 2D). There was an increase of AIMs $(\mathrm{p}<0.05)$ in rats treated chronically with L-DOPA and $\mathrm{H}_{2}$ inhalation produced a reduction of $41 \%$ in LID (Figs. 2B, C).

Animals presenting LID had a decrease in plasma and striatal IL-10 levels in 6-OHDA-lesioned rats when compared with vehicle-treated 6-OHDA-lesioned animals ( $\mathrm{p}<0.05$; Fig. $2 \mathrm{E}$ and J). Interestingly, this effect of L-DOPA was reverted by $\mathrm{H}_{2}$ inhalation ( $\mathrm{p}<0.05$; Fig. $2 \mathrm{E}$ and $\mathrm{J}$ ), indicating that the gas favors this anti-inflammatory cytokine production in samples of LID rats.

L-DOPA treatment caused an increase in striatal pro-inflammatory cytokine IL-1 $\beta$ and IL-6 levels compared to 6-OHDA-lesioned animals treated with vehicle $(\mathrm{p}<0.05$; Fig. $2 \mathrm{~K}$ and $\mathrm{M})$; no statistical differences were observed in plasma (Fig. $2 \mathrm{~F}$ and $\mathrm{H})$. The striatal increase was reverted by $\mathrm{H}_{2}$ inhalation $(\mathrm{p}<0.05)$, indicating that $\mathrm{H}_{2}$ acts not only by up-regulating an anti-inflammatory cytokine, but also by down-regulating those cytokines in LID rats.

There was an enhancement only in striatal TNF- $\alpha$ production that was reverted by $\mathrm{H}_{2}$ inhalation in dyskinetic animals $(\mathrm{p}<0.05$; Fig. $2 \mathrm{~L})$. No differences in plasma or striatal IFN- $\gamma$ levels were found between groups (Fig. 2I and N).

3.3. Cytokines and AIMs correlation To evaluate potential associations between these cytokines and AIMS, we performed a correlation analysis (Fig. 3A). AIMs were negatively correlated to striatal IL-10 (Fig. 3B) and positively correlated to plasma IL-1 $\beta$ (Fig. 3C) and striatal TNF- $\alpha$ levels (Fig. 3D).

Striatal IL-10 levels were negatively correlated to striatal TNF- $\alpha$ levels (Fig. 3E). Plasma IL-1 $\beta$ levels were correlated to striatal and plasma TNF- $\alpha$ levels (Fig. $3 \mathrm{~F}$ and G, respectively). Of note, there is no significant correlation between plasma IL-10 and striatal IL-10, plasma IL-1 $\beta$ and striatal IL-1 $\beta$, and plasma IL-6 and striatal IL-6, indicating disparities in peripheral versus central immune response, even being the same cytokine (Fig. 3A). Other relationships were manifest (see Suppl. Fig. 1 and 2 for details).

3.4. Effects of $\mathbf{H}_{2}$ on striatal astrocyte and microglia on LID Fig. 4A and B show representative immunohistochemical staining of OX-42 and GFAP -ir cells, respectively, in the lesioned striatum of rats with LID under Air or $\mathrm{H}_{2}$ treatment. Regarding striatal OX-42 (Fig. 4C) and GFAP-ir (Fig. 4E) positive cells, an increased number of astrocytes and microglia in the striatum of L-DOPA-treated rats was observed when compared to 6-OHDA-lesioned rats treated with vehicle $(\mathrm{p}<0.05)$. L-DOPA also induced increased changes in astrocyte morphology (processes number and length and intersections number), as indicative of reactive cells. Specifically, the increased intersections mean that the mature astrocyte network has a bigger complexity ${ }^{41} . \mathrm{H}_{2}$ pre-treatment prevented L-DOPA-induced OX-42 increase $(\mathrm{p}<0.05)$, but not GFAP (Figs. $4 \mathrm{C}, \mathrm{E}) . \mathrm{H}_{2}$ prevented the reactivity of striatal microglia of rats with LID, i.e. reducing the number of processes $\left(\mathrm{p}<0.05\right.$, Fig. 4D) and increasing the length of these processes $(\mathrm{p}<0.05$, Fig. $4 \mathrm{G})$. Conversely, $\mathrm{H}_{2}$ caused no change in GFAP morphology compared to the L-DOPA+Air group (Figs. 4F, I, J).

Similarlyto described by Bortolanza et al., $(2015)^{10}$, the increase of the immunoreactivity of GFAP, OX-42 in LID and the evidence of microglia and astrocytes activation was not observed in the striatum of non-lesioned rats and lesioned rats treated only with vehicle or $\mathrm{H}_{2}$ (results not presented).

\subsection{Discussion}

This is the first study to report that $\mathrm{H}_{2}$ reduces LID severity in the PD model. This beneficial effect of $\mathrm{H}_{2}$ inhalation was associated with reduced peripheral and central cytokine levels and microglia activation. Of note, $\mathrm{H}_{2}$ did not interfere with L-DOPA's therapeutic efficacy with motor functioning. 
Recently, the effect of $\mathrm{H}_{2}$ was reported in models of $\mathrm{PD}^{24,25,43}$. Either drinking $\mathrm{H}_{2}$-rich water or intermittent inhalation of $2 \% \mathrm{H}_{2}$ ameliorate 6-OHDA or MPTP-induced hemiparkinsonism ${ }^{37,39}$. The effects of gas inhalation, however, might vary according to the treatment protocol used. An intermittent inhalation of $2 \%$ $\mathrm{H}_{2}$ (15 min; 1-hour interval; 12 hours per day for 5 weeks) ameliorated 6-OHDA-induced hemiparkinsonism rat model, whereas continuous inhalation of $2 \% \mathrm{H}_{2}$ (24 hours a day for 5 weeks) had no effect ${ }^{42}$. As aforementioned, the long-term protocol used and the route of administration of $\mathrm{H}_{2}$ can contribute to maximizing its effects and therefore increase (or not) its benefits. It must be highlighted that the effects observed in the present study were followed by treatment of the animals with a single exposure to $\mathrm{H}_{2}$. The optimal route, time and dose of $\mathrm{H}_{2}$ administration remain to be established.

\subsection{LID inflammatory status in plasma and striatum}

We observed that chronic L-DOPA treatment in the parkinsonian rat was associated with a decrease in plasma and striatum IL-10 levels compared to animals that received its vehicle.

Interestingly, LID was accompanied by an increase in pro-inflammatory markers in the striatum and plasma. Both observations correlate with peripheral and CNS inflammation respectively, after L-DOPA treatment of parkinsonian rats. Previous studies reported some anti-dyskinetic drugs targeting the immune system epitomized by corticosterone ${ }^{44}$, the peroxisome proliferator-activated-gamma agonist rosiglitazone ${ }^{45}$, the immunosuppressant rapamycin ${ }^{45}$, and other drugs with immunomodulatory and antiangiogenic activities such as thalidomide ${ }^{43}$, cannabidiol $^{46-48}$, and nitric oxide synthase inhibitor ${ }^{10}$.

Besides the convincing effect of neuroinflammatory mediators in the onset and development of neurodegenerative disorders and $\operatorname{LID}^{10,44,49,50}$, new studies are now focusing on the role of the peripheral immune system in neurodegenerative disorders as a potential target therapy ${ }^{51-56}$. The peripheral immune system can act directly by brain infiltration ${ }^{57,58}$. Also, microglia are renowned for causing neuroinflammation induced by a peripheral inflammatory stimulus, as clearly observed in classical models of inflammation using lipopolysaccharide as stimulus ${ }^{59}$. Similar to Dos Santos Pereira et $\mathrm{al}^{60}$, here, we showed that plasma IL-1 $\beta$ cytokine levels are concurrently correlated with striatal TNF- $\alpha$ levels and AIMS. Furthermore, striatal TNF- $\alpha$ levels were also correlated with AIMs induced by L-DOPA in the PD model. Collectively, these correlations indicate plasma IL-1 $\beta$ as a potential therapeutic target to treat LID, to be ultimately targeted by other ways besides $\mathrm{H}_{2}$. These correlations suggest a potential effect of plasma IL- $1 \beta$ in AIMs presentation. However, the arising hypothesis still remains elusive and the implicated mechanism must be evaluated in further studies $^{10,11,14,15,37,61}$.

\section{2. $\mathrm{H}_{2}$ and LID: microglia and astrocytes}

Our data provides evidence of the effect of $\mathrm{H}_{2}$ inhalation on decreasing immunohistochemical signal and process number of microglia, along with reduction of their process length in lesioned striatum of rats chronically treated with L-DOPA. The number of intersections could indicate the communication between these cells and we did not find significant differences regarding this criterion. Reactive microglia present macrophagic and antigen-cell function mainly through synthesis of several chemokines and cytokines, inflammatory mediators and cell surface molecules ${ }^{62}$. Otherwise, our results clearly show that $\mathrm{H}_{2}$ inhalation was not able to reduce astrocytes levels back to control levels (rats without L-DOPA treatment). It is important to point out that the consequences of astrogliosis may have both beneficial and detrimental effects on surrounding neural and non-neural cells ${ }^{63}$. Reactive astrocytes change their properties in parallel with morphological, biochemical and functional alterations ${ }^{64-66}$. We also observed that $\mathrm{H}_{2}$ inhalation caused an abrupt decrease in striatal IL-1 $\beta$ and TNF- $\alpha$ cytokines that have important roles in modulating astrocytic phenotypes ${ }^{58}$, and thus supporting a potential shift in reactive astrocyte neurotoxic phenotypes to speculate a more neuroprotective phenotype.

Collectively, our results on the effect of $\mathrm{H}_{2}$ on the inflammatory environment associated with the effects on striatal microglial reactivity may support, at least in part, the reduction of LID. However, we cannot stop speculating on the participation of other mechanisms in this important anti-dyskinetic $\mathrm{H}_{2}$ effect. 
Within the scope of $\mathrm{PD}$, it is known that the levels of reactive oxygen species are strictly controlled by various antioxidant mechanisms in healthy dopaminergic neurons. These effects may be deficient in Parkinson's patients ${ }^{67} .{ }^{68}$. Furthermore, oxidative stress is related to the decline in cell function and apoptosis in several cell types, including astrocytes ${ }^{69}$. Although this was not the study's objective, it is noteworthy that $\mathrm{H}_{2}$ has aroused great interest concerning its antioxidant profile, which is corroborated in diseases and disease models associated with oxidative stress ${ }^{70}$. Such observations may be the target of future investigations.

In addition to oxidative stress, there are several markers for toxicity in the brain, but one of the most widely studied is the neurotransmitter L-glutamate. Although LID is primarily caused by pre-synaptic and postsynaptic changes in DA neurotransmission, it also depends on altered glutamatergic transmission at diverse nodes of the cortico-basal ganglia-thalamocortical network ${ }^{71}$. It could be possible that $\mathrm{H}_{2}$ inhalation action on LID be attributed to affecting neurotransmission systems well directly involved in dyskinesia development, besides its antiinflammatory action. Recently, alleviation of dyskinetic behaviors in both rat and primate models of LID following L-dopa treatment and xenon (another gas) inhalation was documented and this effect is mediated by an improvement of glutamatergic transmission in striatal projecting neurons ${ }^{72}$. In addition, Settineri et al $(2018)^{73}$ evidenced that hydrogenized water protected human neuroblastoma SH-SY5Y cells from oxidative stress and glutamate toxicity.

\subsection{Conclusion}

$\mathrm{H}_{2}$ inhalation decreases abnormal involuntary movements and signaling of an inflammatory reaction in a preclinical L-DOPA-induced dyskinesia rodent model. To our knowledge, no adverse effects of $\mathrm{H}_{2}$ have been reported, and $\mathrm{H}_{2}$ is relatively easy to use, inexpensive, and effective in daily medical practice. Altogether, the search for a compound capable of preventing the side effects of PD without affecting the benefits of the L-DOPA treatment is certainly important to the well-being of patients.

6.0. Acknowledgements We appreciate all the support and guidance provided by Dr. Andrej Romanovsky. The authors are also thankful for the technical assistance of Nadir Fernandes, Mauro F. Silva, and Célia Silva.

\subsection{Authors' Roles}

1) Research project:

A. Conception: BMS, GCN, MB, GTH, LGSB, EDB

B. Organization: BMS, GCN

C. Execution: BMS, GCN, JFP, DSA, MB, LGSB, EDB

2) Statistical Analysis:

A. Design: BMS, GCN, JFP

B. Execution: BMS, GCN, JFP

3) Manuscript writing:

A. Writing of the first: draft: BMS

B. Review and Critique: BMS, GCN, JFP, DSA, MB, GTH, LGSB, EDB

\subsection{Disclosures of all authors (for the preceding 12 months)}

Grant BMS [\#2016/09364-3, São Paulo Research Foundation (FAPESP), Brazil].

Grant MB [\#2016/06602-0, São Paulo Research Foundation (FAPESP), Brazil].

Grant LGSB [\#301040/2018-4, National Council for Scientific and Technological Development (CNPq), Brazil] 
Grant EDB [\#301040/2018-4, National Council for Scientific and Technological Development (CNPq), Brazil], [\#2014/25029-4, São Paulo Research Foundation (FAPESP), Brazil]. Honoraria EDB [Bernice Grafstein Award for Outstanding Accomplishments in Mentoring (SfN-USA)].

\subsection{References}

1. Cenci, M. A. \& Olanow, C. W. Translating scientific advances into disease-modifying therapies for Parkinson's Disease. Experimental Neurology 298, 135-136 (2017). 2. Storch, E. A. et al. The effect of cognitivebehavioral therapy versus treatment as usual for anxiety in children with autism spectrum disorders: A randomized, controlled trial. J. Am. Acad. Child Adolesc. Psychiatry 52, (2013). 3. Angela Cenci, M. Presynaptic mechanisms of L-DOPA-induced dyskinesia: The findings, the debate, the therapeutic implications. Frontiers in Neurology 5, (2014). 4. Cenci, M. A., Riggare, S., Pahwa, R., Eidelberg, D. \& Hauser, R. A. Dyskinesia matters. Mov. Disord. 35, 392-396 (2020). 5. Gomes, M. Z., Raisman-Vozari, R. \& Del Bel, E. A. A nitric oxide synthase inhibitor decreases 6-hydroxydopamine effects on tyrosine hydroxylase and neuronal nitric oxide synthase in the rat nigrostriatal pathway. Brain Res. 1203, 160-169 (2008). 6. Del-Bel, E., Bortolanza, M., Dos-Santos-Pereira, M., Bariotto, K. \& Raisman-Vozari, R. L-DOPA-induced dyskinesia in Parkinson's disease: Are neuroinflammation and astrocytes key elements? Synapse 70, 479-500 (2016). 7. Joers, V., Tansey, M. G., Mulas, G. \& Carta, A. R. Microglial phenotypes in Parkinson's disease and animal models of the disease.Progress in Neurobiology 155, 57-75 (2017). 8. Carta, M. \& Bezard, E. Contribution of pre-synaptic mechanisms to l-DOPA-induced dyskinesia. Neuroscience 198, 245-251 (2011). 9. Porras, G. et al. L-dopa-induced dyskinesia: Beyond an excessive dopamine tone in the striatum. Sci. Rep. 4, 1-5 (2014). 10. Bortolanza, M. et al. Glial activation is associated with l-DOPA induced dyskinesia and blocked by a nitric oxide synthase inhibitor in a rat model of Parkinson's disease. Neurobiol. Dis.73, 377-387 (2015). 11. Mulas, G. et al. Differential induction of dyskinesia and neuroinflammation by pulsatile versus continuous L-DOPA delivery in the 6-OHDA model of Parkinson's disease. Exp. Neurol. 286, 83-92 (2016). 12. Carta, A. R. et al. L-DOPA-induced dyskinesia and neuroinflammation: do microglia and astrocytes play a role? Eur. J. Neurosci. 45, 73-91 (2017). 13. Robelet, S., Melon, C., Guillet, B., Salin, P. \& Kerkerian-Le Goff, L. Chronic L-DOPA treatment increases extracellular glutamate levels and GLT1 expression in the basal ganglia in a rat model of Parkinson's disease. Eur. J. Neurosci. 20, 1255-1266 (2004). 14. Bortolanza, M., Nascimento, G. C. D., Raisman-Vozari R. \& Del-Bel, E. Doxycycline and its derivative, COL-3, decrease dyskinesia induced by L-DOPA in hemiparkinsonian rats. Br. J. Pharmacol. 178, 2595-2616 (2021). 15. Bortolanza, M. et al. Are cyclooxygenase-2 and nitric oxide involved in the dyskinesia of Parkinson's disease induced by L-DOPA?Philos. Trans. R. Soc. Lond. B. Biol. Sci. 370, 20140190 (2015). 16. Nalls, M. A. et $a l$. Large-scale meta-analysis of genome-wide association data identifies six new risk loci for Parkinson's disease. Nat. Genet. 46, 989-993 (2014). 17. Cirstea, M. S. et al. Microbiota Composition and Metabolism Are Associated With Gut Function in Parkinson's Disease. Mov. Disord. 35, 1208-1217 (2020). 18. Westfall, S. et al. Microbiome, probiotics and neurodegenerative diseases: deciphering the gut brain axis. Cellular and Molecular Life Sciences 74, 3769-3787 (2017). 19. Dinan, T. G. \& Cryan, J. F. Gut instincts: microbiota as a key regulator of brain development, ageing and neurodegeneration. J. Physiol. 595, 489-503 (2017). 20. Houser, M. C. \& Tansey, M. G. The gut-brain axis: Is intestinal inflammation a silent driver of Parkinson's disease pathogenesis?npj Parkinson's Disease 3, 1-9 (2017). 21. Scheperjans, F. et al. Gut microbiota are related to Parkinson's disease and clinical phenotype. Mov. Disord.30, 350-358 (2015). 22. Unger, M. M. et al. Short chain fatty acids and gut microbiota differ between patients with Parkinson's disease and agematched controls. Park. Relat. Disord. 32, 66-72 (2016). 23. Ostojic, S. M. Inadequate Production of H2 by Gut Microbiota and Parkinson Disease. Trends in Endocrinology and Metabolism 29, 286-288 (2018). 24. Fujita, K. et al. Hydrogen in drinking water reduces dopaminergic neuronal loss in the 1-methyl-4-phenyl1,2,3,6-tetrahydropyridine mouse model of Parkinson's disease. PLoS One 4, e7247 (2009). 25. Fu, Y. et al. Molecular hydrogen is protective against 6-hydroxydopamine-induced nigrostriatal degeneration in a rat model of Parkinson's disease. Neurosci. Lett. 453, 81-85 (2009). 26. Iketani, M. \& Ohsawa, I. Molecular Hydrogen as a Neuroprotective Agent. Curr. Neuropharmacol. 15, 324-331 (2016). 27. Ohno, K., Ito, M., Ichihara, M. \& Ito, M. Molecular hydrogen as an emerging therapeutic medical gas for neurodegenerative and other diseases. Oxidative Medicine and Cellular Longevity2012, 1-11 (2012). 28. Gao, Q. et al. Molecular 
hydrogen increases resilience to stress in mice. Sci. Rep. 7, 1-12 (2017). 29. Nogueira, J. E. et al. Molecular hydrogen reduces acute exercise-induced inflammatory and oxidative stress status. Free Radic. Biol. Med. 129, 186-193 (2018). 30. Ohsawa, I. et al. Hydrogen acts as a therapeutic antioxidant by selectively reducing cytotoxic oxygen radicals. Nat. Med.13, 688-694 (2007). 31. Nogueira, J. E. et al. Inhaled molecular hydrogen attenuates intense acute exercise-induced hippocampal inflammation in sedentary rats. Neurosci. Lett. 715, 134577 (2020). 32. Saramago, E. A. et al. Molecular hydrogen potentiates hypothermia and prevents hypotension and fever in LPS-induced systemic inflammation. Brain. Behav. Immun. 75, 119-128 (2019). 33. Jesus, A. A. et al. Chronic molecular hydrogen inhalation mitigates short and long-term memory loss in polymicrobial sepsis.Brain Res. 1739, 146857 (2020). 34. Cenci, M. A., Lee, C. S. \& Björklund, A. L-DOPA-induced dyskinesia in the rat is associated with striatal overexpression of prodynorphin- and glutamic acid decarboxylase mRNA. Eur. J. Neurosci.10, 2694-2706 (1998). 35. Lundblad, M. et al. Pharmacological validation of behavioural measures of akinesia and dyskinesia in a rat model of Parkinson's disease. Eur. J. Neurosci. 15, 120-132 (2002). 36. Winkler, C., Kirik, D., Björklund, A. \& Cenci, M. A. L-DOPA-induced dyskinesia in the intrastriatal 6-hydroxydopamine model of Parkinson's disease: Relation to motor and cellular parameters of nigrostriatal function. Neurobiol. Dis. 10, 165-186 (2002). 37. Padovan-Neto, F. E., Echeverry, M. B., Tumas, V. \& Del-Bel, E. A. Nitric oxide synthase inhibition attenuates l-DOPA-induced dyskinesias in a rodent model of Parkinson's disease. Neuroscience159, 927-935 (2009). 38. Cenci, M. A. \& Lundblad, M. Ratings of L-DOPA-Induced Dyskinesia in the Unilateral 6-OHDA Lesion Model of Parkinson's Disease in Rats and Mice. Curr. Protoc. Neurosci. 41, 9-25 (2007). 39. Amorim, M. R. et al. Increased lipopolysaccharideinduced hypothermia in neurogenic hypertension is caused by reduced hypothalamic $\mathrm{PGE}_{2}$ production and increased heat loss. J. Physiol. 598, 4663-4680 (2020). 40. Schneider, C. A., Rasband, W. S. \& Eliceiri, K. W. NIH Image to ImageJ: 25 years of image analysis. Nat. Methods. 9, 671-675 (2012). 41. GiocantiAuregan, A. et al. Altered astrocyte morphology and vascular development in dystrophin-Dp71-null mice. Glia64, 716-729 (2016). 42. Barriola, S., Perez-Cerda, F., Matute, C., Bribian, A. \& Lopez-Mascaraque, L. A Clonal NG2-Glia Cell Response in a Mouse Model of Multiple Sclerosis. Cells 9, 1279 (2020). 43. Ito, M. et al. Drinking hydrogen water and intermittent hydrogen gas exposure, but not lactulose or continuous hydrogen gas exposure, prevent 6-hydorxydopamine-induced Parkinson's disease in rats.Med. Gas Res. 2, 15 (2012). 44. Barnum, C. J. et al. Exogenous corticosterone reduces l-DOPA-induced dyskinesia in the hemi-parkinsonian rat: Role for interleukin-1ß. Neuroscience 156, 30-41 (2008). 45. Santini, E., Heiman, M., Greengard, P., Valjent, E. \& Fisone, G. Inhibition of mTOR signaling in parkinson's disease prevents L-DOPA-induced dyskinesia. Sci. Signal. 2, (2009). 46. dos-Santos-Pereira, M., da-Silva, C. A., Guimarães, F. S. \& Del-Bel, E. Co-administration of cannabidiol and capsazepine reduces L-DOPA-induced dyskinesia in mice: Possible mechanism of action.Neurobiol. Dis. 94, 179-195 (2016). 47. Espadas, I. et al. Beneficial effects of the phytocannabinoid $\triangle 9$-THCV in L-DOPA-induced dyskinesia in Parkinson's disease.Neurobiol. Dis. 141, 104892 (2020). 48. Huot, P., Johnston, T. H., Koprich, J. B., Fox, S. H. \& Brotchie, J. M. The pharmacology of L-DOPA-induced dyskinesia in Parkinson's disease. Pharmacological Reviews 65, 171-222 (2013). 49. Muñoz, A., Garrido-Gil, P., Dominguez-Meijide, A. \& Labandeira-Garcia, J. L. Angiotensin type 1 receptor blockage reduces l-dopa-induced dyskinesia in the 6-OHDA model of Parkinson's disease. Involvement of vascular endothelial growth factor and interleukin-13.Exp. Neurol. 261, 720-732 (2014). 50. Pisanu, A. et al. Neuroinflammation in l-DOPA-induced dyskinesia: beyond the immune function. Journal of Neural Transmission 125, 1287-1297 (2018). 51. Delgado-Alvarado, M. et al. Tau/ $\alpha$-synuclein ratio and inflammatory proteins in Parkinson's disease: An exploratory study.Mov. Disord. 32, 1066-1073 (2017). 52. Fan, Z. et al. Systemic activation of NLRP3 inflammasome and plasma $\alpha$-synuclein levels are correlated with motor severity and progression in Parkinson's disease. J. Neuroinflammation 17, 1-10 (2020). 53. Keegan, A. P. et al. Plasma cytokine IL-6 levels and subjective cognitive decline: preliminary findings. Int. J. Geriatr. Psychiatry 33, 358-363 (2018). 54. Lai, K. S. P. et al. Peripheral inflammatory markers in Alzheimer's disease: A systematic review and meta-analysis of 175 studies. J. Neurol. Neurosurg. Psychiatry 88, 876-882 (2017). 55. Li, D., Song, X., Huang, H., Huang, H. \& Ye, Z. Association of Parkinson's disease-related pain with plasma interleukin-1, interleukin-6, interleukin-10, and tumour necrosis factor- $\alpha$.Neurosci. Lett. 683, 181-184 (2018). 56. Morgan, A. R. et al. The correlation between inflammatory biomarkers and polygenic risk score in Alzheimer's disease. J. Alzheimer's Dis. 56, 25-36 (2017). 57. Neuroimmune communication. 
Nature Immunology 18, 115 (2017). 58. Tan, E. K. et al. Parkinson disease and the immune system associations, mechanisms and therapeutics. Nature Reviews Neurology 16, 303-318 (2020). 59. Juan, Z. et al. Oral administration of Clostridium butyricum CGMCC0313-1 reduces ovalbumin-induced allergic airway inflammation in mice. Respirology 22, 898-904 (2017). 60. Dos Santos Pereira, M. et al.Contributive Role of TNF- $\alpha$ to L-DOPA-Induced Dyskinesia in a Unilateral 6-OHDA Lesion Model of Parkinson's Disease. Front. Pharmacol. 11, 2204 (2021). 61. Padovan-Neto, F.E., Cavalcanti-Kiwiatkoviski, R., Carolino, R.O.G., Anselmo-Franci, J., Del Bel, E. Effects of prolonged neuronal nitric oxide synthase inhibition on the development and expression of l-DOPA-induced dyskinesia in 6-OHDA-lesioned rats.Neuropharmacology 89, 87-97 (2015). 62. Reemst, K., Noctor, S. C., Lucassen, P. J. \& Hol, E. M. The indispensable roles of microglia and astrocytes during brain development. Front. Hum. Neurosci. 10, 566 (2016). 63. Hamby, M. E. \& Sofroniew, M. V. Reactive Astrocytes as Therapeutic Targets for CNS Disorders. Neurotherapeutics 7, 494-506 (2010). 64. Hol, E. M. \& Pekny, M. Glial fibrillary acidic protein (GFAP) and the astrocyte intermediate filament system in diseases of the central nervous system. Curr. Opin. Cell Biol. 32, 121-130 (2015). 65. Parpura, V. et al. Glial cells in (patho)physiology. Journal of Neurochemistry 121, 4-27 (2012). 66. Sofroniew, M. V. \& Vinters, H. V. Astrocytes: Biology and pathology. Acta Neuropathologica 119, 7-35 (2010). 67. Puspita, L., Chung, S. Y. \& Shim, J. W. Oxidative stress and cellular pathologies in Parkinson's disease. Mol. Brain.10, 53 (2017). 68. Schildknecht, S. et al. Oxidative and nitrative alphasynuclein modifications and proteostatic stress: implications for disease mechanisms and interventions in synucleinopathies. Journal of Neurochemistry 125, 491-511 (2013). 69. Amri, F., Ghouili, I., Amri, M., Carrier, A. \& Masmoudi-Kouki, O. Neuroglobin protects astroglial cells from hydrogen peroxide-induced oxidative stress and apoptotic cell death. Journal of neurochemistry 140, 151-169 (2017). 70. Huang, C. S., Kawamura, T., Toyoda, Y. \& Nakao, A. Recent advances in hydrogen research as a therapeutic medical gas. Free Radical Research 44, 971-982 (2010). 71. Sgambato-Faure, V. \& Cenci, M. A. Glutamatergic mechanisms in the dyskinesias induced by pharmacological dopamine replacement and deep brain stimulation for the treatment of Parkinson's disease.Progress in Neurobiology, 96, 69-86 (2012). 72. Baufreton, J. et al. Inhaling xenon ameliorates l-dopa-induced dyskinesia in experimental parkinsonism. Movement Disorders 33, 1632-1642 (2018). 73. Settineri, R. et al. Hydrogenized water effects on protection of brain cells from oxidative stress and glutamate toxicity. Am. J. Food Nutr. 6, 9-13 (2018).

Figures: (Up to 5)

Figure 1. Molecular hydrogen $\left(\mathrm{H}_{2}\right)$ inhalation reduces AIMS without interfering with the antiparkinsonian effect of L-DOPA. (A, B) Schematic protocol. After Sham surgery or 6-OHDA injection, the rats were analysed in the apomorphine-induced rotational test for lesion confirmation. Then, Sham or 6-OHDA-lesioned rats were treated chronically (15 days) with L-DOPA or its vehicle. Subsequently before the last L-DOPA injection, Air mixture or $\mathrm{H}_{2}$ inhalation was performed for a 1-h period, followed by the LDOPA injection and abnormal involuntary movements on axial, limb and orofacial parameters (ALO AIMs) and locomotor activity were measured. (C) 6-OHDA lesion significantly increased total contralateral turns induced by apomorphine. (D) Photomicrographs of coronal brain sections illustrating the loss of TH positive immunolabeling in the striatum fibers (D) and substantia nigra compacta neurons (E), scale bars $=1200 \mu \mathrm{m}$. $(\mathrm{F}, \mathrm{G}) \mathrm{H}_{2}$ treatment does not alter the L-DOPA effect on the distance traveled $(\mathrm{F})$ and rearing $(\mathrm{G})$ in the actimeter. (H) Time course of the appearance of dyskinetic manifestation with 6-OHDA+L-DOPA+Air mixture compared to 6-OHDA+L-DOPA $+\mathrm{H}_{2}$ inhalation $(\mathrm{p}<0.001)$ across time (for each 20 -min period). (I, $\mathrm{J}, \mathrm{K}$ ) show the co-administration of L-DOPA and $\mathrm{H}_{2}$ inhalation effects on the axial (I), limb (J) and orofacial (K) AIMs over 120 mins. Sham rats treated with vehicle of L-DOPA (SHAM+Veh, white); Sham rats treated chronically with L-DOPA (SHAM+L-DOPA, orange); 6-OHDA lesioned rats treated with vehicle of L-DOPA (6-OHDA+Veh, green); 6-OHDA lesioned rats treated chronically with L-DOPA (6-OHDA+L-DOPA, blue). AIMs were analyzed using 2-Way RM ANOVA analysis with Tukey's multiple comparisons post-hoc test. Distance travelled was analyzed by ordinary One-Way ANOVA with Fisher post-hoc test. Data are reported as mean \pm SEM. ${ }^{* * *} \mathrm{p}<0.001,{ }^{* *} \mathrm{p}<0.01,{ }^{*} \mathrm{p}<0.05$.

Figure 2. Molecular hydrogen $\left(\mathrm{H}_{2}\right)$ inhalation improves peripheral and striatal inflammation in L-DOPA-treated 6-OHDA-lesioned rats. (A) Schematic timeline for cytokine measurements. (B) 
Time course of the appearance of dyskinetic manifestation of the animals used for cytokines measurement (6$\mathrm{OHDA}+$ Veh+Air mixture; 6-OHDA+L-DOPA+Air mixture; 6-OHDA+L-DOPA $+\mathrm{H}_{2}$ inhalation; $\left.\mathrm{p}<0.001\right)$ across time (for each 20-min period). (C) show the co-administration of L-DOPA and $\mathrm{H}_{2}$ inhalation effects on the sum of axial, limb and orofacial AIMs over 120 mins of these animals. (D) These animals were tested also in the apomorphine test, 6-OHDA lesion significantly increased total contralateral turns induced by apomorphine. Plasma and striatal levels of IL-10 (E and J), IL-1 $\beta$ (F and K), TNF- $\alpha$ (G and L), IL-6 (H and $\mathrm{M}$ ) and IFN- $\gamma$ (I and N) 2 hours after L-DOPA administration in rats inhaling $\mathrm{H}_{2}\left(\mathrm{~L}-\mathrm{DOPA}+\mathrm{H}_{2}\right)$ or not (L-DOPA + Air). 6-OHDA-lesioned rats were represented by the purple bars; 6-OHDA-lesioned rats treated chronically with L-DOPA inhaling Air were represented by the blue bars, and 6-OHDA-lesioned rats treated chronically with L-DOPA and inhaling $\mathrm{H}_{2}$ were represented by the green bars. $\mathrm{H}_{2}$ inhalation caused an increase in plasma and striatal levels of IL-10 $(\mathrm{p}<0.05)$ and a decrease in plasma and striatal levels of IL-1 $\beta(\mathrm{p}<0.05)$, striatal levels of TNF- $\alpha(\mathrm{p}<0.05)$ and plasma and striatal levels of IL-6 $(\mathrm{p}<0.05)$ in L-DOPA treated rats. All cytokine measurements were statistically analyzed by ordinary One-Way ANOVA with Fisher's post-hoc test. Bars are represented as mean \pm SEM. \# p $<0.05$ vs. L-DOPA + Air (blue bars), ${ }^{*} \mathrm{p}<0.05$ vs. Veh + Air (purple bars) groups.

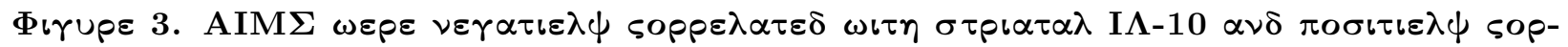

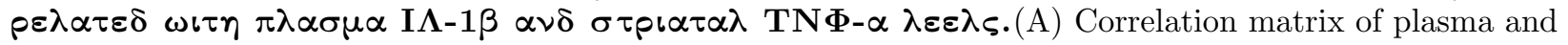
striatal IL-10, IL-1 $\beta$, TNF- $\alpha$ levels, and AIMs. The correlation coefficient (r) of each correlation is inside its correspondent box ranging from -1 (darkest red gradient) to +1 (darkest blue gradient). A two-tailed p-value was chosen to evaluate the statistical significance of each correlation. The coefficient of determination $\left(\mathrm{r}^{2}\right)$ between a statistically correlated pair of variables was calculated and a best-fit linear regression line (dark full line in the plot) was built for each two variables in one panel (panels B-G). Correlation plot was set at 95\% confidence interval (shadowed area with the exactly gradient of the correspondent correlation coefficient - r) surrounded by dark dashed lines. There was a statistical correlation between: AIMS and striatal IL-10 levels (B); AIMS with plasma IL-1 $\beta$ (C) and AIMs with striatal TNF- $\alpha$ (D) levels; striatal TNF- $\alpha$ levels with striatal IL-10 (E), plasma IL-1 $\beta$ with striatal TNF- $\alpha(\mathrm{F})$, and plasma TNF- $\alpha(\mathrm{G})$ levels.

Figure 4. Molecular hydrogen $\left(\mathrm{H}_{2}\right)$ inhalation prevented the reactivity of striatal microglia with no impact in striatal astrocytes morphology. Microglia analysis by OX-42-ir staining. (A) representative photomicrographs of striatum slices of lesioned rats treated chronically with L-DOPA inhaling (L-DOPA $+\mathrm{H}_{2}$ ) or not (L-DOPA) $\mathrm{H}_{2}$. Quantification of OX-42-ir images indicates the number of OX-42-ir cells (B), total number of processes cells (C), average processes length (D), and intersections number between the processes $(\mathrm{G}) . \mathrm{H}_{2}$ treatment induced a decrease in the number of OX42-ir positive cells, in the average of processes number and length, a without affecting the number of intersections in L-DOPA-treated rats (Fig. $\mathrm{H} ; \mathrm{p}>0.05$ ). Astrocyte analysis by GFAP staining. (B) representative photomicrographs of striatum slices of 6-OHDA-lesioned rats treated chronically with L-DOPA inhaling (L-DOPA $+\mathrm{H}_{2}$ ) or not (L-DOPA) $\mathrm{H}_{2}$. Quantification of GFAP images indicates the number of GFAP cells (E), the total number of processes cells $(\mathrm{F})$, average processes length (I), and intersections number between the processes $(\mathrm{J}) . \mathrm{H}_{2}$ treatment caused no alterations in the number of GFP positive cells, processes number, length, and intersections ( $\mathrm{p}>0.05)$. 6-OHDA-lesioned rats were represented by the purple bars; 6-OHDA-lesioned rats treated chronically with L-DOPA inhaling Air was represented by the blue bars, and 6-OHDA-lesioned rats treated chronically with L-DOPA and inhaling $\mathrm{H}_{2}$ were represented by the green bars. Data were statistically analyzed by ordinary One-Way ANOVA with Fisher post-hoc test. Bars are represented as mean \pm SEM. \#p $<0.05$ vs. L-DOPA + Air, ${ }^{*} \mathrm{p}<0.05$ vs. Veh + Air groups. 